\title{
Foundational ontologies in action
}

\author{
Understanding foundational ontology through examples
}

\author{
Stefano Borgo ${ }^{\mathrm{a}}$, Antony Galton ${ }^{\mathrm{b}}$ and Oliver Kutz ${ }^{\mathrm{c}, *}$ \\ ${ }^{a}$ LOA Laboratory for Applied Ontology, ISTC, National Research Council, Trento, Italy \\ E-mail: stefano.borgo@cnr.it \\ ${ }^{\mathrm{b}}$ University of Exeter, $U K$ \\ E-mail: apgalton@exeter.ac.uk \\ ${ }^{\mathrm{c}}$ KRDB Research Centre for Knowledge and Data, Department of Computer Science, \\ Free University of Bozen-Bolzano, Bolzano, Italy \\ E-mail: Oliver.Kutz@unibz.it
}

Keywords: Foundational ontologies, upper ontologies, BFO, DOLCE, GFO, GUM, TUpper, UFO, YAMATO

\section{Motivations for and development of this special issue}

The idea of proposing a special issue on the use of foundational ontologies for modelling simple everyday, or commonsense, situations came after several discussions with people in different domains which pointed to difficulties in understanding the philosophical and general presentations in the documentation accompanying foundational systems. This problem, we noticed, is worsened by the lack of step-by-step guidance to model a real situation from the point of view of a specific ontology. The result was that different knowledge engineers could generate mutually inconsistent models starting from the very same foundational ontology even when working on the same topic. Given that one of the main roles of ontologies is to serve as interoperability drivers, this conclusion was puzzling if not discouraging.

We observed that it would not be possible to provide solutions to these issues without involving the very teams that developed foundational ontologies. On the other hand, if we could collect a series of presentations centred around the presentation of modelling cases, we would also have fostered a more consistent use of foundational ontologies, and set the bases for practical and comparative evaluations of the consequences in adopting one ontology rather than another.

Another contribution that a special issue on "Foundational Ontologies in Action" could provide, and whose consideration eventually led to the particular selection of use cases discussed in this special issue, is to indicate how these ontologies differ in modelling core aspects in knowledge engineering practices. Such core aspects include the description of artefacts and their components, the modelling of changes

\footnotetext{
${ }^{*}$ Corresponding author. E-mail: Oliver.Kutz@unibz.it.
} 
of an object's properties, and the representation of changes in social situations. All these cases show up in a variety of ways in standard modelling activities but, from the ontological viewpoint, all follow just a few modelling patterns. Once these modelling patterns are made evident, we concluded, the comparison of these ontologies for practical applications will also be easier.

Eventually, the systematic modelling scenarios discussed in this special issue should make it possible for the knowledge engineer to select the right ontology without the need to dig into sophisticated philosophical dilemmas and discussions. This said, we insist that in using a foundational ontology one has to have a basic understanding of the philosophical choices that motivate a particular system, but also think that, in practice, it is sufficient that this encompasses an idea of the practical consequences of those philosophical choices which, we hope, is what the papers in this special issue are providing.

\section{A brief history of the FOUST project}

The ideas sketched above began to take concrete shape in 2017 when Stefano Borgo (Trento) and Oliver Kutz (Bolzano) organised the first FOUST 2017 workshop, which was held on February 27 and 28 at the KRDB Research Center of the Free University of Bozen-Bolzano, Italy. This event was already organised with the goal in mind to bring together some of the core designers of the most used foundational ontologies with a strong philosophical underpinning. The acronym 'FOUST', derived from 'The FOUndational STance', here was a word-play that on the one hand alluded to Daniel Dennett's 'intentional stance ${ }^{11}$ which, roughly, considers the behaviour of entities in terms of mental properties (Dennett, 1987), and on the other, by contrast, to the 'foundational stance' of Guarino's ontological level, where the intended meaning of a modelling language is formally constrained by requiring predicates to satisfy meaning postulates (Guarino, 1994, 2009).

The FOUST 2017 workshop eventually invited some of the ontologists involved in the design of the following systems, in alphabetical order: BFO, DOLCE, GFO, GUM, UFO, and YAMATO. Later during the development of the FOUST project, also the TUpper ontology was invited to participate, resulting in the final list of seven ontological systems that participate in this special issue. Invited participants of the first FOUST meeting included: John Bateman, Antony Galton, Nicola Guarino, Giancarlo Guizzardi, Heinrich Herre, Frank Loebe, Claudio Masolo, Riichiro Mizoguchi, Fabian Neuhaus, João Nicola, and Barry Smith. ${ }^{2}$ The goal and eventual outcome of this workshop was to discuss the concrete use-cases that could be employed to illustrate and elaborate the differences between the popular foundational systems in addressing basic modelling problems, and the logical means that should be employed. At this stage, the discussion also revolved around using different logical languages for a common formalisa-

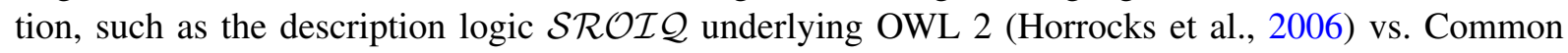
Logic $^{3}$ (Mossakowski et al., 2014) vs. more standard first-order logic (Kutz et al., 2010). Moreover, we discussed approaches and proof methods to establishing formal consistency results for the ontological systems, similarly to how they were pursued earlier for the consistency proof of DOLCE (Kutz and Mossakowski, 2011). In this context, it was also considered to what extent the systems could be aligned and uniformly presented in a logical structuring language for ontologies such as the 'Distributed Ontology, Modelling, and Specification Language' (DOL) standardised by the OMG (Object Management

\footnotetext{
${ }^{1}$ Dennett's 'intentional stance' is the third and final level in a 3-level hierarchy of 'physical-design-intentional'.

${ }^{2}$ See https://foust.inf.unibz.it/foust1/.

${ }^{3}$ The ISO document for the Common Logic language is freely available at this link: https://www.iso.org/standard/66249. html.
} 
Group, 2016; Mossakowski et al., 2012). ${ }^{4}$ A language such as DOL would comprise all of the above logical languages, and additionally provide the technical means to structure, align, and formally compare the systems.

However, both meta-(onto)logical questions such as the consistency of the systems as well as a more uniform and structured formal presentation of the systems had to be left for future efforts. Instead, we decided to focus on the modelling of use cases, and the eventual outcome of this workshop was to settle on the basic structure of the template for the papers presented in this special issue, which is briefly summarised in Section 4. Furthermore, it was decided to use standard first-order logic in the papers to maximise the readability and comparability of the modellings presented.

After the first FOUST meeting it became clear that the workshop addressed an important gap in the ecosystem of Applied Ontology as a scientific field. Subsequently, the FOUST workshops became an important venue for presenting and publishing work on all aspects of foundational ontology, including comparisons of different foundational approaches, extensions and alignments between them, formal and logical work (such as consistency proofs), as well as examples of the application of foundational ontology in areas such as AI, Semantic Web, Linguistics, and so on.

As of 2022, FOUST is in its sixth edition, following the first workshop in Bolzano in $2017 .{ }^{5}$ FOUST I was organised as part of the Joint Ontology Workshops (JOWO), FOUST II in 2017 in Bolzano (Italy), FOUST III 2019 in Graz (Austria), FOUST IV and V in 2020 and 2021 in Bolzano (Italy) as part of the 2020-21 Bolzano Summer of Knowledge (BOSK), ${ }^{6}$ and FOUST VI in Jönköping (Sweden). ${ }^{7}$ The series is now organised under the auspices of IAOA, the International Association for Ontology and its Applications (iaoa.org), and has formally established a steering committee.

\section{Participating ontologies}

As mentioned above, the FOUST workshops resulted in the creation of a template to discuss ontological modelling cases (presented below) and the invitation of seven foundational ontologies to model those cases, namely: BFO (Otte et al., 2022), DOLCE (Borgo et al., 2022), GFO (Loebe et al., 2022), GUM (Bateman, 2022), TUpper (Grüninger et al., 2022), UFO (Guizzardi et al., 2022), and YAMATO (Mizoguchi and Borgo, 2022). We here give a brief characterisation of their origin and development much more complete historical data about each of the ontologies can be found in the papers of this special issue.

BFO. 'BFO' stands for 'Basic Formal Ontology'. It is a top-level ontology designed to support information integration, retrieval, and analysis across all domains of scientific investigation, and has been in development since the BFO project began around 2002 (Smith et al., 2004). It serves as the top-level ontology of the Open Biomedical and Bioinformatic Ontology (OBO) Foundry and the Industrial Ontology Foundry (IOF). BFO is part of the standard ISO 21838 (part 2) where it is axiomatised in Common Logic (CLIF) and in the Web Ontology Language (OWL).

\footnotetext{
${ }^{4}$ The OMG specificaton is available at the following link: https://www.omg.org/spec/DOL/.

${ }^{5}$ See https://foust.inf.unibz.it/.

${ }^{6}$ See https://summerofknowledge.inf.unibz.it.

${ }^{7}$ The proceedings of all FOUST workshops are published in the IAOA series at CEUR, see http://ceur-ws.org/iaoa.html. The volumes for FOUST II-V can be found at the following links: http://ceur-ws.org/Vol-2050/, http://ceur-ws.org/Vol-2518/, http://ceur-ws.org/Vol-2708/, http://ceur-ws.org/Vol-2969/.
} 
DOLCE. 'DOLCE' stands for 'Descriptive Ontology for Linguistic and Cognitive Engineering'. Its first comprehensive development goes back to work carried out at ISTC-CNR (Trento \& Rome) for the European project 'WonderWeb'8 in the early 2000s, published in Masolo et al. (2003). The axiomatisation of this version used FOL and additionally some modalities. Following this work, DOLCE has been applied in a number of areas and several application-oriented "lite" versions were published axiomatised in 'lightweight' versions of OWL, including DOLCE-lite, DOLCEultralite, and DOLCE-zero. DOLCE is part of the standard ISO 21838 (part 3) where it is axiomatised in Common Logic (CLIF) and in the Web Ontology Language (OWL).

GFO. 'GFO' stands for 'General Formal Ontology'. Work on GFO arose from research on formal ontology at the University of Leipzig starting in 1999, aiming to achieve a better foundation for knowledge representation in the field of medicine. The initial system was called 'General Ontological Language' (GOL) (Degen et al., 2001) and developed into the current GFO system (Herre et al., 2006). Its primary formalisation is in (type-free) FOL. Additionally, a few parts are represented in OWL. GFO has mainly been applied in industry-related research projects on medical tasks (risk management, navigation in surgery, metadata and search) and biomedical topics (cell tracking, representing phenotypes and functions).

GUM. 'GUM' stands for 'Generalized Upper Model'. Its current version goes back to ontological work begun in the context of natural language processing AI projects in the 1980s, specifically the Penman 'Upper Model' (Bateman et al., 2010). In addition to natural language processing, GUM has been applied in spatial reasoning and cognitive robotics, and formalised versions have been developed both in OWL and in a sorted first-order logic, namely the CASL language. ${ }^{9}$

TUpper. 'TUpper' stands for 'A Top Level Ontology within Standards'. The TUpper Ontology has developed quite recently since around 2017 (Grüninger et al., 2017) and is designed as a top-level ontology that contains modules from ontologies within existing international standards, extended so as to satisfy the criteria for top level ontologies specified in ISO/IEC 21838-1. The goal of TUpper is to support the ontological analysis of relevant existing standards and to integrate the ontologies within those standards. TUpper is part of the standard ISO 21838 (part 4) where it is axiomatised in Common Logic (CLIF) and in the Web Ontology Language (OWL).

UFO. 'UFO' stands for the 'Unified Foundational Ontology'. It is a top-level ontology that has been being developed for almost two decades and is designed to provide foundations for Conceptual Modelling and Data Semantics in Computer Science. ${ }^{10}$ UFO has been extensively used in government, industry, and academia, and in a variety of domains, chiefly in Software Engineering, but also in social, legal and economic domains. UFO also served as top-level ontology for the OntoUML conceptual modeling language and its tool ecosystem. UFO has been formalised in first-order modal logics with type reification, specified in the TPTP syntax.

YAMATO. 'YAMATO' stands for 'Yet Another More Advanced Top-level Ontology'. It was developed around 2005 and was first named 'Yet Another Top-level Ontology', abbreviated 'YATO', which means 'finally' in Japanese. The current name was suggested by Peter Simons, after the Japanese

\footnotetext{
${ }^{8}$ See http://wonderweb.man.ac.uk/index.shtml.

${ }^{9}$ See http://www.informatik.uni-bremen.de/cofi/index.php/CoFI; CASL is closely related to the DOL language discussed above in terms of its structuring capabilities, see https://www.omg.org/spec/DOL/.

${ }^{10}$ See https://nemo.inf.ufes.br/en/projetos/ufo/.
} 
battleship Yamato, and it is also the name of the old country of Japan. YAMATO was designed with both engineering and philosophy in mind, and is implemented in the ontology editor Hozo. ${ }^{11}$

\section{Historical perspectives}

Historically, the stimulus for developing ontologies has come from a number of different disciplines, notably Philosophy, Linguistics, Cognitive Science, and Computer Science. Of these, Philosophy has the deepest roots, extending back millennia, and many topics traditionally treated within Metaphysics we would now recognise as essentially ontological. Some of these will be discussed in the next section. In developing ontologies for application to information systems, however, researchers have often taken pains to draw a line between what they are doing and philosophical ontology, on the grounds that the goals of the two enterprises are quite different, with applied ontology answerable to pragmatic concerns supposedly absent from purely philosophical pursuits. We see the contrast already in Gruber's wellknown declaration that "[a]n ontology is an explicit specification of a conceptualization" (Gruber, 1993), which he immediately follows with the observation that "[t]he term is borrowed from philosophy, where an ontology is a systematic account of Existence". Nonetheless, in developing foundational ontologies handling highly general concepts with application across many, or all, specific subject matters, applied ontologists have inevitably found themselves addressing precisely the same issues as the philosophers.

Within Computer Science, in particular, a major role in the development of applied ontologies has been played by the sub-discipline of Artificial Intelligence, and within that, the areas of Knowledge Representation and Knowledge Engineering. Here, work on foundational issues has been of key importance for top-level ontologies, even when authors have not explicitly labelled their work as ontological. Prominent examples include work on the ontology of time (Allen, 1984), space (Randell et al., 1992; Borgo et al., 1996), motion (Galton, 1993), and causality, all topics that play essential roles in practical tasks such as planning, prediction, and explanation. A major concern in these areas has been the development of systematic approaches to "commonsense" reasoning, as set out for example in the Naïve Physics project which was instigated by Patrick Hayes (Hayes, 1979, 1985) and left its mark on much subsequent work in Knowledge Representation (Hobbs and Moore, 1985; Davis, 1990, 2008).

These topics are also of key importance for natural language processing, which can involve both understanding and generation of natural language texts. Hence linguistics has also been a stimulus for the development of high-level ontological thinking. Ontological concerns in linguistics are particularly prominent in the areas of Semantics, which is concerned with meaning, and Cognitive Linguistics with its concern for how meanings are represented in the mind. Here too we find an interest in such topics as time (Moens and Steedman, 1988; Pustejovsky, 1991; Mani et al., 2005), space (Talmy, 1983; Bloom et al., 1996; Bateman et al., 2010), and parthood (Winston et al., 1987), amongst others.

In truth it would be invidious draw sharp boundaries between the different disciplines involved here. Much work in this area is interdisciplinary, drawing on philosophical, linguistic, cognitive, or computational resources as appropriate, and discussion of topics such as those mentioned above can be found in all these disciplines. Anyone wishing to research the classification of occurrents, for example, would do well to consult the literature in philosophy (Vendler, 1957; Galton, 1984), linguistics (Mourelatos, 1978; Moens and Steedman, 1988), and artificial intelligence (Allen, 1984), as well as more recent work in applied ontology such as Galton and Mizoguchi (2009).

\footnotetext{
${ }^{11}$ See http://www.hozo.jp/ for the Hozo editor. Hozo and OWL files of YAMATO are freely available for use and can be found at http://www.hozo.jp/onto_library/upperOnto.htm.
} 


\section{Philosophical issues}

The previous section briefly outlined how the origins of Foundational Ontology can be traced back to a number of distinct disciplines. Although the initial developments within these disciplines were somewhat independent of each other, they all picked up on a range of philosophical issues that have been debated for thousands of years - Aristotle in particular being an enduring influence. Amongst such issues, a number stand out as especially relevant to modern work in foundational ontology; here we have no space to do much more than merely to list them with a few brief comments.

(1) Continuants vs Occurrents. This is the distinction between entities such as material objects that endure through time, undergoing various kinds of change as they do so, and entities such as events that unfold over time, manifesting as changes in continuants but not themselves undergoing change. In the Aristotelian tradition continuants are treated as primary while occurrents, if thought of as entities at all, are regarded as dependent upon them. A persistent undercurrent of processoriented thinking, ultimately traceable to Heraclitus and reflected in the work of more recent thinkers such as Whitehead, Bergson, Broad, Sellars, Rescher, Seibt, and Dupré, seeks to reverse the order of dependence. Throughout the history of philosophy, though, the Aristotelian position has had the upper hand, and this is reflected in most modern upper ontologies. ${ }^{12}$

(2) Universals vs Particulars. All ontologies recognise the distinction between terms denoting individuals and terms denoting generic covering entities by which individuals may be counted as in some way the "same" - whether these be classes, containing individuals as members (e.g., the class of all cats), concepts by which we group together individuals sharing a salient set of common properties (e.g., the idea of cats in general), or some hard-to-define notion of categories, kinds, or universals to which particular individuals are related as instances (the generic cat). The point of disagreement, however, is what status to assign to these generic entities - are they elements of the world described by the ontology, or do they only exist within the ontology itself, as part of the technical paraphernalia by which it describes that world? This was already a topic of concern in mediaeval philosophy, where it showed up as the debate between Realists, for whom universals were real-world entities, and Nominalists, for whom they were merely labels, or concepts, used to impose an intelligible order on reality but not themselves part of that reality. The debate is alive and kicking in modern ontology too, as witness the debate between realism and conceptualism played out in the recent exchange of articles (Merrill, 2010a; Smith and Ceusters, 2010; Merrill, 2010b).

(3) Concrete vs Abstract. It is common to distinguish between concrete entities such as continuants and occurrents, which exist in space and/or time, and abstract entities which, if they can be said to exist at all, do so outside space and time, prototypical examples being mathematical entities such as numbers, functions, and sets. Opinions differ as to whether abstractions are part of mindindependent reality or cognitive artefacts, and as a result some ontologies (e.g., DOLCE) include a category of abstracts in their taxonomy, whereas others (e.g., BFO) do not. Ontologies of the former kind treat times and places, for example, as abstract entities, whereas for those of the latter, they are regarded as concrete, times being occurrents and places continuants.

\footnotetext{
${ }^{12}$ But there are exceptions, for example BORO (de Cesare and Partridge, 2016), which does not distinguish between continuants and occurrents, treating both uniformly as four-dimensional entities with extension in both space and time - this is the metaphysical stance of perdurantism (Lewis, 1986).
} 
(4) Substance vs Accident. In classical logic, an entity's properties are expressed by means of predicates such as "... is red", where the dots represent the fact that, as Frege held, predicates are "unsaturated". This means that a predicate only expresses a thought when combined with a term denoting an entity that may or may not have the property expressed by the predicate. ${ }^{13}$ Here properties are not themselves entities and so have no place within an ontology's taxonomy. But this is not the only possible approach. Instead of seeing "Mars is red", in Fregean fashion, as the result of applying the predicate "... is red" to the name "Mars" (where the latter, but not the former, denotes an individual entity), we could rather see it as the result of applying a relational predicate "... is ..." to two entity-denoting expressions "Mars" and "red", so that the thought is of a relation holding between the entities they denote. To complete this picture, though, we must specify what kind of entity "red" denotes, and various possibilities have been explored in the literature. One is that "red" denotes a trope - the individual redness of Mars itself, as distinct from that of, say, the star Antares, even if these are qualitatively identical. Another is to take "red" to denote a universal, the same redness featuring in the thoughts expressed by "Mars is red" and "Antares is red". A third possibility, used in several ontologies, invokes entities called "qualities", unique to individuals, such as, for example the colour of Mars and the colour of Antares, and then takes "red" to denote a value that such a quality can assume.

\section{The FOUST template}

As discussed in the introductory remarks above, the contributions to the FOUST special issue follow a common structure. A common template was initially proposed by Borgo \& Kutz for the first FOUST workshop in 2017, then jointly discussed at the workshop and eventually finalised after further feedback and refinements. The final FOUST template, followed by all contributions, has the following core structure, where we use $O$ to denote generically some foundational ontology:

Introduction: An introduction to the ontology, together with some historical background and motivations.

Section 1. "Principles and structure of ontology O": This section gives the ontological background and ontological criteria that are at the core of the ontology, as well as introducing the ontology's categories and the properties and relations it uses.

Section 2. "The formalisation of $O$ ": This section discusses the overall formalisation approach. Focus is on those parts of the ontology used in the cases, and on typical axiomatisation patterns.

Section 3. "Analysis and formalisation in O: examples": This section presents how to formalise the given cases in the ontology.

Section 4. "Ontology usage and community impact": This section gives information on usage and application domains.

Appendix: This appendix summarises the notation in three columns: 'ontological entity (categories, relations)', 'formal expression', 'description in natural language'.

The core of each paper, of course, is Section 3, which analyses ontologically, using $O$, the use cases we agreed on to model. The six example use cases are briefly listed below, pointing out for each firstly a

\footnotetext{
${ }^{13}$ And similarly, mutatis mutandis, for relational predicates with more than one argument place.
} 
'GOAL', providing the general motivation for its inclusion in terms of the group of modelling problems that it targets, and secondly a 'FOCUS', that is, a target group of specific ontological issues.

Brief overviews and a partial comparison of how the different ontologies approach the modelling of these examples are then provided in the remaining sections of this introduction.

\section{Case 1: (Section 3.1. Composition/constitution)}

"There is a four-legged table made of wood. Some time later, a leg of the table is replaced. Even later, the table is demolished so it ceases to exist although the wood is still there after the demolition."

GoAL: The example aims to show if and how the ontology models materials, objects, and components and the relationships among them.

Focus: The relationship between the wood and the table and the table's parts over time. (Artefacts and functions are not the focus.)

\section{Case 2: (Section 3.2. Roles)}

"Mr. Potter is the teacher of class $2 C$ at Shapism School and resigns at the beginning of the spring break. After the spring break, Mrs. Bumblebee replaces Mr. Potter as the teacher of 2C. Also, student Mary left the class at the beginning of the break and a new student, John, joins in when the break ends."

GoAL: The example aims to show if and how the ontology models the relationships between roles, players and organisations.

Focus: The change of roles/players; the vacancy of the teaching position; persistence of the class while students come and go.

\section{Case 3 (a): (Section 3.3. Property change)}

"A flower is red in the summer. As time passes, the colour changes. In autumn the flower is brown."

GoAL: The example aims to show if and how the ontology models change in qualities/properties.

Focus: The change of the colour of an object.

\section{Case 3 (b): (Section 3.3. Property change)}

"A man is walking when suddenly he starts walking faster and then breaks into a run."

GOAL: The example aims to show if and how the ontology models change during an event.

Focus: The change in the speed and mode of locomotion.

Case 4: (Section 3.4. Event change)

"A man is walking to the station, but before he gets there, he turns around and goes home."

GOAL: The example aims to show if and how the ontology models change in goal-directed activities.

Focus: An activity/event is not completed and another activity/event is completed instead.

\section{Case 5: (Section 3.5. Concept evolution)}

BACKGROUND: A marriage is a contract between two people that is present in most social and cultural systems and it can change in major (e.g. gender constraints) and minor (e.g. marriage breaking procedures) aspects.

"A marriage is a contract that is regulated by civil and social constraints. These constraints can change but the meaning of marriage continues over time."

GoAL: The example aims to show if and how the ontology models the evolution of the meaning of a term.

Focus: The continuity/discontinuity of the meaning of marriage in the presence of changing qualifications. 


\section{Comparing foundational ontologies in action}

\subsection{Constitution and composition: Tables and cut-off legs}

The first scenario that we consider in the template is as follows:

"There is a four-legged table made of wood. Some time later, a leg of the table is replaced. Even later, the table is demolished so it ceases to exist although the wood is still there after the demolition."

Since an ontology is not bound to model a description at the literal level, each ontology is free to make sense of what is described following its own perspective. That is, what is formally modelled by each ontology is an ontological reading of the above scenario. In many cases, and this one is among them, an ontology may be compatible with different 'interpretations' of the given description, leading to the construction of distinct models, each one compatible and aligned with that ontology.

For the constitution scenario, here are some possibilities that could be adopted

(1) There are objects (like table, legs) and these are distinct from matter (like wood). What the scenario describes are changes in the relationships these entities satisfy (like the constitution, parthood, component relationships).

(2) There are objects (like material entities) which play some roles (like table-role, legs-role). ${ }^{14}$ The players of the roles can change over time. The scenario describes changes in the relationships these entities satisfy (like the play relationship).

(3) There is a material object that has functionalities (like table-function) and so do some of its parts (like leg-function). The scenario describes changes in the material object and its functionalities.

(4) There are objects (as in (1)-(3)) and there are events. The scenario describes the event during which there are changes of the relationships among these objects.

(5) There is a process, that includes (sub)processes, which presents relevant regularities (like tableprocess, leg-process). The process and its subprocesses evolve manifesting changes while maintaining the overall regularities while they exist.

Most ontologies in this special issue can be used to develop models according to more than one of these general views.

\subsection{Roles: The school teacher and the student}

In some cases an entity has a special position because of the context in which it is involved. When the entity is an agent, this position is often enriched with special duties and rights. In these cases the entity may be identified via dedicated names like 'teacher' (for persons), 'engine' (for devices) and 'living room' (for spaces). These special positions are known as roles; most foundational ontologies include a theory of roles to help model these cases.

The second use case of this special issue is about roles and their formal treatment in ontology. Roles can be of different types, e.g., functional roles (relative to goal achievement), structural roles (relative to components of systems) and organisational roles (relative to social systems).

The use case considered in the special issue is about a social scenario with two types of roles. One, the teacher role, can be played by one person at a time. The second one, student, can be played by several people at the same time. The scenario includes a period in which there is no teacher. The scenario is described as follows:

\footnotetext{
${ }^{14}$ For roles, see next subsection.
} 
"Mr. Potter is the teacher of class $2 \mathrm{C}$ at Shapism School and resigns at the beginning of the spring break. After the spring break, Mrs. Bumblebee replaces Mr. Potter as the teacher of 2C. Also, student Mary left the class at the beginning of the break and a new student, John, joins in when the break ends."

In some approaches, a role is a dependent entity that exists only when played by some other entity (e.g., when roles are taken as 'realisable entities', like dispositions, as in BFO). In other views, roles may exist also when no entity is playing them (e.g., when roles are taken as concepts as in DOLCE and YAMATO). In our scenario, the approach adopted by the different ontologies is made evident by looking at how each ontology models the teacher role during the time in which the position is vacant. Finally, sometimes an ontology allows one to talk about an entity as player of a role, e.g., a person-as-client in a business relationship. In the literature, this special kind of entity is known as a qua-entity.

\subsection{Qualities of objects and qualities of events}

\subsubsection{Qualities of objects: The colour of the flower}

The seven ontologies exhibit a variety of different treatments of the case of the flower changing colour, albeit with many commonalities. All the ontologies, naturally enough, recognise the flower as an individual entity, but when it comes to its colour there is no consensus as to how to handle it. In standard first-order logic the usual approach would be to treat ascription of redness to the flower as a matter of simple predication, using the form Red(flower), and of the ontologies presented here only TUpper follows this approach. The next simplest alternative is to treat 'red' as a noun rather than an adjective, and possession of a colour as a relation between the coloured object and the colour it has, along the lines of HasColour(flower, red). None of the ontologies here does exactly this, but they do accept the idea that red, or redness, is a kind of entity to which a red thing stands in some relation. A further level of sophistication is to regard the colour of the flower as an entity distinct from either the flower itself or its redness. The colour is an entity specifically dependent on the flower, and at different times it may assume different values. Logically, we might crudely characterise this position as HasColour(flower, colour) $\wedge$ HasValue (colour, red, $t$ ). The differences we see in the ontologies reflect various different approaches to how this should be articulated - depending crucially on what kind of entity red is taken to be.

The second main difference we see concerns the approach to representing change. Traditionally, logical analysis has favoured what might be called a 'snapshot' view, according to which there is nothing to change over and above the holding of different static states ('snapshots') at different times. Against this, many have argued that changes - represented by, for example, processes or events - should be regarded as some kind of entities in their own right, and indeed most of the ontologies here do indeed include a category of occurrents or perdurants to put them in. Despite this, the analyses offered by the papers collected here are for the most part content to represent the change in colour of the flower in terms of the succession of two states of the world, in one of which the flower is red, and in the other, brown.

For the purpose of giving an overview of the differences and similarities in how the ontologies describe this scenario, we here concentrate on five points of comparison, as follows. We gloss over details, for which the reader is referred to the accounts of the individual ontologies, but here provide a brief summary for the purposes of comparison. It should be emphasised that this synopsis only indicates what is shown in the actual analyses presented by the individual papers, and should not be taken to imply any limitations as to what alternative analyses might be possible within the same ontologies. 
(1) Are qualities such as colour reified, that is, are they represented as entities within the ontology? This is essentially the difference, at the ontological rather than the linguistic level, between using 'red', say, as a noun or as an adjective. All of the ontologies represented here apart from TUpper do reify qualities.

(2) Is the individual colour of the flower reified, as an entity inhering in the flower and distinct both from the flower and its value and from the colour of any other flower? All the ontologies apart from GUM and TUpper reify the individual colour of the flower.

(3) How is the value of the individual colour of the flower (if reified) related to whatever is denoted by the names such as 'red' and 'brown' we use to describe them? The different ontologies exhibit a variety of approaches here, the relationship in question depending on what the references of colour names are taken to be. For BFO and GFO, they are universals and the relationship is 'instance of'; for DOLCE, they are regions and the relationship is 'part of'; for UFO they are classes and the relationship is 'member of'; for YAMATO the relationship given is identity.

(4) How are these items denoted 'red' and 'brown' related to a broader structure such as a colour space within which they are defined? For BFO 'red' and 'brown' denote subclasses of 'colour' whereas for DOLCE they are parts of a colour space and for UFO they are members of a class of colour concepts. GFO states that the universal 'red', of which the value of the individual flower colour may be an instance, is part of the "value structure" for measuring and depicting colours, but the nature of this value structure is not further specified. In DOLCE, by contrast, with a colour space corresponding to GFO's value structure, it is clear that, as in item (3), the parthood relation here is that of subregion.

(5) Is the event or process of change modelled explicitly or only implicitly through the contrast between start and end states? Both GUM and TUpper clearly model the changing process itself, as opposed to defining it by its start and endpoints. In the BFO analysis, a process of change is explicitly modelled, but the change in question is the withering of the flower rather than its change in colour; but there is no reason to suppose that this treatment could not be extended to the latter change as well. Other ontologies offer a variety of ways to model this change. YAMATO models the change as a transition between start and end states but it could also represent colour change as an ongoing process. DOLCE even provides a formula to ensure that the change is continuous across the colour space.

\subsubsection{Qualities of events: The speed of the walk}

All the ontologies model the described scenario as an occurrence with three consecutive phases: a 'walking' phase, a 'walking faster' or "accelerating" phase, and a 'running' phase (although GUM specifically focusses on the 'breaking into a run' which initiates the running). Most of the ontologies take these three phases to be parts of the overall occurrence, designated 'proper occurrent parts' or the like. The man participates in these parts and hence in the whole occurrence. Within this broad characterisation the ontologies exhibit significant differences in the details of their treatment.

A key point concerns how an ontology characterises the three phases. Some of the ontologies (BFO, GFO) label the parts as 'Walking', 'Fast walking' (or 'accelerating'), and 'Running' without attempting to define the differences between these. In justification of this, the authors of the GFO paper point out that the fine-grained classification of human locomotion types is difficult and as yet unsolved. 
Other ontologies attempt to characterise the difference between the phases in terms of some quality of the activities involved, ${ }^{15}$ for example speed (DOLCE, GUM, UFO), though it is recognised that the difference between walking and running is not just one of speed. For DOLCE, Speed Quality is a subclass of Temporal Quality, which covers the qualities of perdurants (occurrents). UFO likewise assigns "Speed Values" to locomotion events, but these values are not further defined beyond 'walking', 'walking fast', and 'running'. For GUM, speed, like colour, is a 'sense and measure quality', to which an eventuality stands in a relation manner - it is a feature of the manner in which the eventuality takes place.

TUpper takes a somewhat different approach, treating each of the phases as itself subdivided into a sequence of individual leg-movements, which for walking are called 'steps', and for running, 'leaps'. The difference between walking and fast walking is defined by the duration of each step, being less in the latter than in the former; here duration is a value asigned to an occurrence.

In all these cases the event and its parts are treated as occurrents. As such, any properties ascribed to them are immutable: they belong to the occurrent as a whole and cannot be regarded as changing over time. This makes it difficult to model the continuous increase in speed which presumably characterises the second stage of the scenario - and indeed the treatments given for TUpper, GUM, and UFO model the second stage just as fast walking rather than acceleration. ${ }^{16}$ The remaining ontology, YAMATO, takes a very different approach, drawing a sharp distinction between events, which are classical occurrents as just described, and processes, which are more continuant-like in the sense that they can change while unfolding. For YAMATO, an event such as described in the present scenario is constituted by one or more processes, and in analysing the scenario here the focus is on these constitutive processes: a walking process present on the first two subintervals and a running process present at the end of these (and presumably for a while thereafter). The speed is a quality that inheres in the processes rather than in the man participating in them. Over the first two subintervals this has a walking speed value, which is constant over the first subinterval and monotonically increasing over the second. At the end, once the running process has begun, the speed value must be a running speed value, though this is not explicitly stated in the analysis.

\subsection{Events and their goals: Changing destination while going somewhere}

The scenario features a man walking to the station but returning home before he gets there. This could be modelled purely physically, without reference to the man's plans or intentions: he walks from home to a point somewhere on the route to the station, turns round, and walks home again. But such an analysis misses the point: to say the man is walking to the station implies he intends to get there. That he turns round and goes home before fulfilling this intention amounts to a change of plan, one intention replaced by another that is then successfully realised. This requires an additional layer of analysis. All the analyses offered recognise in some way the importance of this additional factor, but only GFO makes the layered structure explicit, describing the physical aspect separately before introducing goals and goal achievements.

Of the other analyses, those of BFO, DOLCE, and YAMATO explicitly refer to plans. For BFO a plan is a concretisation of a plan specification whose proper parts include action and objective specifications.

\footnotetext{
${ }^{15}$ This approach would not be possible for BFO, in which only continuants - and hence not occurrents - are allowed to have qualities.

${ }^{16}$ Admittedly, the original description of the scenario does not explicitly mention acceleration, so it is compatible with this description to take the increased speed of the second phase to be constant.
} 
The former prescribes a process in which the bearer of the plan (the man) may participate over some interval; this process may or may not achieve the specified objective. In the given scenario the initial plan (walk to the station) is replaced by a new plan (return home) before its objective is achieved.

The YAMATO analysis is broadly similar, the main difference being that processes are treated as ongoing entities capable of undergoing change as they progress. A plan is understood as a representation of a sequence of actions; it is executed by performing a sequence of actions satisfying it, but may be abandoned if only part of the sequence is performed. In the modelled scenario, a walking process is present over an interval. Over some initial subinterval, this process constitutes an uncompleted execution of the plan to walk to the station. After that the process changes so that during an immediately succeeding subinterval it constitutes a turning event. This is followed by a final subinterval over which the process constitutes a completed execution of a plan to go home.

In DOLCE a plan is modelled as a concept, which is a non-physical endurant. An event executes a plan if it complies with the plan's requirements; this does not imply the plan is executed in its entirety. In the given scenario, there are two plans, to walk to the station and to walk home; the whole event is divided into three subevents, the walk towards the station, the turn, and the walk home. The first subevent executes the first plan and the second two together execute the second. The analysis does not include the fact that the execution of the first plan is incomplete whereas that of the second is complete, although this could be added.

Instead of plans, the UFO analysis uses the walker's intentions, via the notion of intended destination. A walking event cannot change, so the analysis uses an associated endurant Walk which encapsulates the walker's intention with his capabilities qua walker. In the given scenario, Walk changes from an 'ongoing walk', in which the destination has not yet been reached, to a 'finalised walk', in which it has. Moreover, a finalised walk may be either a 'successful walk', meaning that the destination arrived at is the originally intended one, or a 'redirected walk', in which it is different as a result of the walker's change of intention. It is the latter kind that is presented in this scenario.

In the GUM analysis, each walking event is modelled as having a destination which associates a location with the event. The semantic modelling specifies the condition to be fulfilled for the event to be achieved (the destination reached), but makes no commitment to its being achieved. That the walk to the station is not achieved is indicated by the present progressive form ('is walking') together with the phrase "before he gets there"; the non-progressive form of 'goes home', indicates that this event is achieved - although neither of these inferences from the verb forms appears to be explicitly shown in the formalisations.

Finally, the TUpper analysis presents the scenario as one where one of two things could happen: either the man continues all the way to the station or he turns round and goes home before getting there. The only formal axiom given states that the turning round occurs in every occurrence of the journey, so that what actually happens is the second possibility. This highlights the fact that what happens is one of a pair of possibilities; although the analysis does not refer to plans or intentions, we could perhaps take this to indicate that since the turn and return home happens rather than the full walk to the station, there has been a change of plan.

\subsection{Social concepts: The marriage and its understanding across time}

Social reality relies on conventions, rules and customs which are sometimes coded in the language and made explicit in laws. Social concepts tend to evolve in time to adapt to changes in the social system. Due to its normative nature, law tends to fix the meaning of the social concepts it addresses; call these the 
legal concepts. The fifth use case of the FOUST special issue aims to show how foundational ontology can model the relationship between social and legal concepts and how concepts can evolve over time.

The description of the use case captures this tension between the social concept and the legal concept:

"A marriage is a contract that is regulated by civil and social constraints. These constraints can change but the meaning of marriage continues over time."

The use case description is accompanied with a clarification on how to understand the case: marriage is a contract between two people that is present in most social and cultural systems and it can change in major (e.g., gender constraints) and minor (e.g., marriage breaking procedures) aspects. Of course, the actual classification of marriage's aspects as major or minor is culture-dependent, yet the examples suffice to clarify the purpose of the use case.

Depending on the ontological view, one can decide to read this case in different ways. In one case, for instance, the ontology recognises that a term persists in the language but not the meaning it denotes. In another reading, the ontology takes the meaning as persisting even though some regulatory aspects of it may change as time goes on. Another way to tackle the use case is by distinguishing the intensional vs. extensional content of the concept: the intensional content of a concept is the set of properties (including their relationships) that defines the concept, the extensional content is the set of instances of the concept.

This brief description shows that this use case admits several interpretations and formal models. Generally speaking, the ontologies in this volume allow alternative views which are discussed at length in the introductory text that each ontology provides. The reader can check further the details of these modellings in the various contributions in this volume.

\section{This is it}

This FOUST special issue at Applied Ontology was five years in the making, but we believe the joint efforts and coordination of seven groups of ontologists has paid back in the end. This introduction could only provide a glimpse of the wealth of detail provided in the papers of this special issue. With this volume, however, we indeed now have a detailed set of case studies and ontological modellings to hand that should allow theorists as well as practitioners to have fast-track access to the different modelling approaches and paradigms proposed and supported by the leading foundational ontologies.

\section{Acknowledgements}

Stefano Borgo was partially supported by the European project OntoCommons (GA 958371). We also thank the Free University of Bozen-Bolzano and the Province of South-Tyrol (Italy) for a generous financial sponsoring of the first two FOUST workshops.

\section{References}

Allen, J. (1984). Towards a general theory of action and time. Artificial Intelligence, 23, 123-154. doi:10.1016/0004-3702(84) 90008-0.

Bateman, J.A. (2022). GUM: The generalized upper model. Applied Ontology, 17(1), 107-141. doi:10.3233/AO-210258.

Bateman, J.A., Hois, J., Ross, R. \& Tenbrink, T. (2010). A linguistic ontology of space for natural language processing. Artificial Intelligence, 174, 1027-1071. doi:10.1016/j.artint.2010.05.008. 
Bloom, P., Peterson, M., Nadel, L. \& Garrett, M. (Eds.) (1996). Language and Space. Cambridge, MA: MIT Press.

Borgo, S., Ferrario, R., Gangemi, A., Guarino, N., Masolo, C., Porello, D., Sanfilippo, E.M. \& Vieu, L. (2022). DOLCE: A descriptive ontology for linguistic and cognitive engineering. Applied Ontology, 17(1), 45-69. doi:10.3233/AO-210259.

Borgo, S., Guarino, N. \& Masolo, C. (1996). A pointless theory of space based on strong connection and congruence. In L.C. Aiello, J. Doyle and S. Shapiro (Eds.), Principles of Knowledge Representation and Reasoning: Proceedings of the Fifth International Conference (KR'96) (pp. 220-229). San Francisco, CA: Morgan Kaufmann.

Davis, E. (1990). Representations of Commonsense Knowledge. San Mateo, CA: Morgan Kaufmann.

Davis, E. (2008). Pouring liquids: A study in commonsense physical reasoning. Artificial Intelligence, 172, 1540-1578. doi:10. 1016/j.artint.2008.04.003.

de Cesare, S. \& Partridge, C. (2016). BORO as a foundation to enterprise ontology. Journal of Information Systems, 30(2), 83-112. doi:10.2308/isys-51428.

Degen, W., Heller, B., Herre, H. \& Smith, B. (2001). GOL: Toward an axiomatized upper-level ontology. In N. Guarino, B. Smith and C. Welty (Eds.), Proceedings of the 2nd International Conference on Formal Ontology in Information Systems, FOIS 2001 (pp. 34-46). ACM.

Dennett, D.C. (1987). The Intentional Stance. Cambridge: The MIT Press.

Galton, A. (1984). The Logic of Aspect: An Axiomatic Approach. Oxford: Clarendon Press.

Galton, A. (1993). Towards an integrated logic of space, time, and motion. In R. Bajcsy (Ed.), Proceedings of the Thirteenth International Joint Conference on Artificial Intelligence (IJCAI'93) (pp. 1550-1555). San Mateo, CA: Morgan Kaufmann.

Galton, A. \& Mizoguchi, R. (2009). The water falls but the waterfall does not fall: New perspectives on objects, processes and events. Applied Ontology, 4(2), 71-107. doi:10.3233/AO-2009-0067.

Gruber, T.R. (1993). A translation approach to portable ontology specifications. Knowledge Acquisition, 5, 199-220. doi:10. 1006/knac.1993.1008.

Grüninger, M., Chui, C. \& Katsumi, M. (2017). Upper ontologies in COLORE. In Proceedings of the Joint Ontology Workshops (JOWO). CEUR Workshop Proceedings. CEUR-WS.org.

Grüninger, M., Ru, Y. \& Thai, J. (2022). TUpper: A top level ontology within standards. Applied Ontology, 17(1), 143-165. doi:10.3233/AO-220263.

Guarino, N. (1994). The ontological level. In R. Casati, B. Smith and G. White (Eds.), Philosophy and the Cognitive Sciences. Proc. of the 16th Wittgenstein Symposium. Kirchberg, Austria, Vienna, August 1993 (pp. 443-456). HölderPichler-Tempsky.

Guarino, N. (2009). The ontological level: Revisiting 30 years of knowledge representation. In A. Borgida, V. Chaudhri, P. Giorgini and E. Yu (Eds.), Conceptual Modelling: Foundations and Applications. Essays in Honor of John Mylopoulos (pp. 52-67). Springer. doi:10.1007/978-3-642-02463-4_4.

Guizzardi, G., Botti Benevides, A., Fonseca, C.M., Porello, D., Almeida, J.P.A. \& Prince Sales, T. (2022). UFO: Unified Foundational Ontology. Applied Ontology, 17(1), 167-210. doi:10.3233/AO-210256.

Hayes, P.J. (1979). The naïve physics manifesto. In D. Michie (Ed.), Expert Systems in the Micro-Electronic Age (pp. 242-270). Edinburgh University Press.

Hayes, P.J. (1985). Naïve physics I: Ontology for liquids. In J.R. Hobbs and R.C. Moore (Eds.), Formal Theories of the Commonsense World (pp. 71-107). Norwood, NJ: Ablex Publishing Corporation.

Herre, H., Heller, B., Burek, P., Hoehndorf, R., Loebe, F. \& Michalek, H. (2006). General Formal Ontology (GFO) - A foundational ontology integrating objects and processes [Version 1.0]. Onto-Med Report 8, Research Group Ontologies in Medicine, Institute of Medical Informatics, Statistics and Epidemiology, University of Leipzig, Germany.

Hobbs, J.R. \& Moore, R.C. (Eds.) (1985). Formal Theories of the Commonsense World. Norwood, NJ: Ablex Publishing Corporation.

Horrocks, I., Kutz, O. \& Sattler, U. (2006). The even more irresistible $\mathcal{S R O} \mathcal{I} \mathcal{Q}$. In Proc. of the 10th Int. Conf. on Principles of Knowledge Representation and Reasoning (KR2006) (pp. 57-67). AAAI Press.

Kutz, O. \& Mossakowski, T. (2011). A modular consistency proof for DOLCE. In Twenty-Fifth Conference on Artificial Intelligence (AAAI-11), San Francisco, CA: AAAI Press.

Kutz, O., Mossakowski, T. \& Lücke, D. (2010). Carnap, Goguen, and the hyperontologies: Logical pluralism and heterogeneous structuring in ontology design. Logica Universalis, 4(2), 255-333. Special Issue on 'Is Logic Universal?'. doi:10.1007/ s11787-010-0020-3.

Lewis, D. (1986). On the Plurality of Worlds. New York: Blackwell.

Loebe, F., Burek, P. \& Herre, H. (2022). GFO: The General Formal Ontology. Applied Ontology, 17(1), 71-106. doi:10.3233/ AO-220264.

Mani, I., Pustejovsky, J. \& Gaizauskas, R. (Eds.) (2005). The Language of Time: A Reader. Oxford, UK: Oxford University Press.

Masolo, C., Borgo, S., Gangemi, A., Guarino, N. \& Oltramari, A. (2003). WonderWeb Deliverable D18: Ontology Library. Technical report, ISTC-CNR. 
Merrill, G.H. (2010a). Ontological realism: Methodology or misdirection? Applied Ontology, 5(2), 79-108. doi:10.3233/AO2010-0076.

Merrill, G.H. (2010b). Realism and reference ontologies: Considerations, reflections and problems. Applied Ontology, 5(3-4), 189-221. doi:10.3233/AO-2010-0080.

Mizoguchi, R. \& Borgo, S. (2022). YAMATO: Yet-Another More Advanced Top-level Ontology. Applied Ontology, 17(1), 211-232. doi:10.3233/AO-210257.

Moens, M. \& Steedman, M. (1988). Temporal ontology and temporal reference. Computational Linguistics, 14, 15-28. Reprinted in Mani et al. 2005.

Mossakowski, T., Codescu, M., Kutz, O., Lange, C. \& Gruninger, M. (2014). Proof support for Common Logic. In Proc. of the Workshop on Automated Reasoning for Quantified Non-classical Logic (ARQNL), July 23, Vienna Summer of Logic.

Mossakowski, T., Lange, C. \& Kutz, O. (2012). Three semantics for the core of the Distributed Ontology Language. In M. Donnelly and G. Guizzardi (Eds.), 7th International Conference on Formal Ontology in Information Systems (FOIS). Frontiers in Artificial Intelligence and Applications (Vol. 239, pp. 337-352). IOS Press. FOIS Best Paper Award.

Mourelatos, A.P.D. (1978). Events, processes, and states. Linguistics and Philosophy, 2(3), 415-434. doi:10.1007/BF00149015.

Object Management Group (2016). The Distributed Ontology, Modeling, and Specification Language (DOL). Document Number ptc/2016-02-37. Available at http://www.omg.org/spec/DOL.

Otte, J., Beverley, J. \& Ruttenberg, A. (2022). BFO: Basic Formal Ontology. Applied Ontology, 17(1), 17-43. doi:10.3233/AO220262.

Pustejovsky, J. (1991). The syntax of event structure. Cognition, 41, 47-81. doi:10.1016/0010-0277(91)90032-Y.

Randell, D.A., Cui, Z. \& Cohn, A.G. (1992). A spatial logic based on regions and connection. In B. Nebel, C. Rich and W. Swartout (Eds.), Principles of Knowledge Representation and Reasoning: Proceedings of the Third International Conference (KR'92) (pp. 165-176). San Mateo, CA: Morgan Kaufmann.

Smith, B. \& Ceusters, W. (2010). Ontological realism: A methodology for coordinated evolution of scientific ontologies. Applied Ontology, 5(3-4), 139-188. doi:10.3233/AO-2010-0079.

Smith, B., Grenon, P. \& Goldberg, L. (2004). Biodynamic ontology: Applying BFO in the biomedical domain. Studies in Health and Technology Informatics, 102, 20-38.

Talmy, L. (1983). How language structures space. In H. Pick and L. Acredolo (Eds.), Spatial Orientation: Theory, Research, and Application (pp. 225-282). New York: Plenum Press. doi:10.1007/978-1-4615-9325-6_11.

Vendler, Z. (1957). Verbs and times. The Philosophical Review, 66(2), 143-160. doi:10.2307/2182371.

Winston, M.E., Chaffin, R. \& Herrmann, D. (1987). A taxonomy of part-whole relations. Cognitive Science, 11, 417-444. doi:10.1207/s15516709cog1104_2. 